\section{RICYDE. Revista Internacional de Ciencias del Deporte} doi: $10.5232 /$ ricyde

Rev. int. cienc. deporte

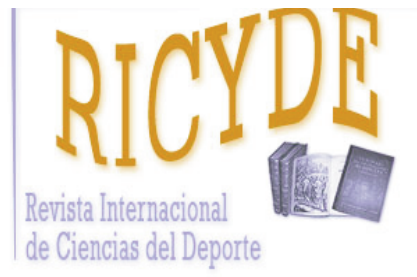

RICYDE. Revista Internacional de Ciencias del Deporte VOLUMEN XIII - AÑO XIII

Páginas:88-103 ISSN:1885-3137

Número 48 - Abril - 2017

\title{
Efectos en el rendimiento físico a corto plazo de dos programas de entrenamiento neưromuscular con diferente orientación aplicados en jugadores de fútbol de élite U-17
}

\section{Short-term physical performance effects of two different neuromuscular oriented training programs on U-17 elite soccer players}

\author{
Javier Raya-González ${ }^{1,2}$, Luis Suárez-Arrones ${ }^{1}$, Manuel Moreno-Puentedura², \\ Jesús Ruiz-Márquez ${ }^{2}$ y Eduardo Sáez de Villarreal ${ }^{1}$
}

1.Universidad Pablo de Olavide, Sevilla. España

2.Córdoba C.F., Departamento de Preparación Física. España

\section{Resumen}

El objetivo de este trabajo fue comparar el efecto de dos programas de entrenamiento neuromuscular (orientación vertical vs horizontal), realizados durante 6 semanas, sobre el rendimiento físico en jugadores de fútbol. Dieciséis futbolistas de 16,6 $\pm 0,3$ años fueron asignados de manera aleatoria en dos grupos experimentales que complementaron su entrenamiento habitual de fútbol con un programa de entrenamiento neuromuscular dos días a la semana. El entrenamiento del grupo vertical ( $n=8, \mathrm{GV}$ ) consistió en los ejercicios $1 / 2$ sentadilla y squat lateral realizados en un dispositivo inercial, y salto vertical al cajón, y el entrenamiento del grupo horizontal $(n=8, G H)$ estuvo basado en ejercicios resistidos de sprint lineal y con cambio de dirección (COD). Antes y después del periodo de intervención se midió la altura de salto vertical, el tiempo de sprint lineal y COD y la potencia del tren inferior. Se obtuvieron mejoras sustanciales (probables a muy probables) en GV en salto con contramovimiento (CMJ) [TE: 0,49, VC: Probable (93/7/0\%)], test de Abalakov (ABK) [TE: 0,38, VC: Probable (83/16/1\%)] y velocidad media propulsiva (VMP) en todas las cargas utilizadas, además de empeorar el tiempo de sprint lineal y COD hacia la izquierda (COD-I). El GH mejoró sustancialmente el tiempo de sprint $10 m$ $[$ TE = 0,63, VC: Muy Probable (98/2/0\%)], COD hacia la derecha (COD-D) $[$ TE = 0,36, VC: Probable (76/23/1\%)], COD-I [TE = 0,34 , VC: Probable (80/19/1\%)], y VMP con 15-30-50kg, además de una posible mejora en el tiempo de sprint en 20 y $30 \mathrm{~m}$. El análisis inter-grupos mostró mejoras sustanciales mayores en GV en CM] [TE = 0,39, VC: Muy Probable (76/22/0\%)], y en GH en tiempo de sprint en 10-20-30m y COD hacia la derecha (COD-D). Los resultados obtenidos sugieren que el entrenamiento del GH es más efectivo en la optimización de la condición física específica del futbolista.

Palabras clave: capacidad de salto; velocidad media propulsiva; sobrecarga excéntrica; sprint lineal; cambio de dirección.

\section{Abstract}

The aim of this research was to compare the effects of two different neuromuscular training programs (vertical vs. horizontal orientation), conducted during 6 weeks, on the physical performance of soccer players in-season. Sixteen soccer players of $16.6 \pm 0.3$ years were randomly assigned to two equally large experimental groups, and both supplemented the soccer training with a specific neuromuscular training program 2 days/week. Vertical group's training $(n=8, V G)$ was composed by half-squat using a flywheel device and vertical jump to box and horizontal group's training $(n=8, H G)$ consisted on linear and non-linear resisted sprints. Before and after the training period jumping ability, linear and non-linear sprint and lower-limb power were measured. Substantial improvements (likely to very likely) were found in the VG in countermovement jump (CMJ) (ES: 0.49), Abalakov test (ABK) (ES: 0.38) and Mean Propulsive Velocity (all load measured), in addition to decrease the performance in linear and non-linear sprint (COD-L). The HG improved substantially in 10m sprint (Effect size [ES]: 0.63), COD-R (ES: 0.36), COD-L (ES: 0.34) and in Mean Propulsive Velocity in $15-30-50 \mathrm{~kg}$. Furthermore, a possible improvement in 20 and $30 \mathrm{~m}$ sprint was also reported in HG. Between-groups analysis showed greater substantial improvements in VG in CMJ (ES: 0.39), and in HG in 10-20-30m sprint and COD-R. The results obtained suggest that horizontal group's training program could be more effective in order to optimize the specific physical condition of the soccer players.

Key words: jumping ability; mean propulsive velocity; eccentric-overload; linear sprint; change-of-direction. 
Raya-González, J.; Suárez-Arrones, L.; Moreno-Puentedura, M.; Ruiz-Márquez, J., y Sáez de Villareal, E. (2017). Efectos en el rendimiento físico a corto plazo de dos programas de entrenamiento neuromuscular con diferente orientación aplicados en jugadores de fútbol de élite U-17. RICYDE. Revista internacional de ciencias del deporte, 48(13), 88-103.

\section{Introducción}

$\mathrm{E}$ 1 fútbol es un deporte intermitente (Di Salvo, Baron, Tschan, Calderon-Montero, Bachl, y Pigozzi, 2007) donde acciones de alta intensidad como sprints, saltos y cambios de dirección, pueden llegar a ser factores determinantes para conseguir el éxito deportivo, tanto en futbolistas adultos (Reilly, Bangsbo y Franks, 2000) como en jóvenes (Castagna, D’Ottavio y Abt, 2003). Además, los desplazamientos a alta intensidad y este tipo de acciones durante la competición se han visto incrementadas en los últimos años (Bush, Barnes, Archer, Hogg y Bradley, 2015), por lo que es esencial tenerlas en cuenta a la hora de programar el entrenamiento de fútbol (Hoff y Helgerud, 2004).

Para mejorar la capacidad de realizar las acciones de alta intensidad que se suceden durante el juego, el entrenamiento de fuerza cobra un papel fundamental en la optimización del rendimiento (Cronin y Hansen, 2005). Con el fin de obtener estas mejoras en los futbolistas, se han aplicado principalmente programas de fuerza constituidos por ejercicios orientados prioritariamente en el plano vertical, los cuales han mostrado efectos positivos en jugadores de fútbol sobre la capacidad de salto vertical (Thomas, French y Hayes, 2009; Chelly, Fathloun, Cherif, Ben Amar, Tabka, y Van Praagh, 2009; Wong, Chamari y Wisloff, 2010), la reducción del tiempo de sprint en diferentes distancias (Chelly y col., 2009; Sander, Keiner, Wirth, y Schmidtbleicher, 2013; Wong y col., 2010) y el aumento de fuerza y/o potencia del tren inferior (Chelly y col., 2009; Sander y col., 2013). Otras variables relacionadas con el rendimiento en fútbol como la agilidad (Thomas y col., 2009), la velocidad del balón tras golpeo, la distancia recorrida en test intermitentes o la economía de carrera (Wong y col., 2010) también se han visto mejoradas debido al efecto de este tipo de programas de fuerza. En esta línea, Thomas y col. (2009) utilizaron ejercicios pliométricos dos veces a la semana durante seis semanas como trabajo adicional al entrenamiento de fútbol, obteniendo mejoras significativas en la agilidad y capacidad de salto vertical. Sander y col. (2013) propusieron un entrenamiento de fuerza basado en ejercicios tradicionales (front squat y back squat) en jugadores jóvenes durante dos años, mejorando estos su fuerza dinámica máxima y el tiempo en sprint en $30 \mathrm{~m}$. Por otro lado, Gorostiaga, Izquierdo, Ruesta, Iribarren, González-Badillo, e Ibáñez. (2004) combinaron en su trabajo ejercicios tradicionales y pliométricos, consiguiendo tras 11 semanas de entrenamiento mejoras significativas en la altura de salto con contramovimiento (CMJ) sin carga y con cargas de 20 y $30 \mathrm{~kg}$, reduciendo también el tiempo de sprint en $5 \mathrm{~m}$.

También se han utilizado, aunque en menor medida, programas de fuerza basados en ejercicios orientados prioritariamente en el plano horizontal. Meylan y Malatesta. (2009) llevaron a cabo un programa de fuerza con mayor orientación horizontal (ejercicios pliométricos) en jugadores jóvenes y durante 8 semanas, obteniendo mejoras en el tiempo de sprint y agilidad, además de incrementar la capacidad de salto vertical. En la misma línea, Marques, Pereira, Reis, y van den Tillaar. (2013) administraron en futbolistas jóvenes un entrenamiento pliométrico combinado con diferentes ejercicios de sprint. Tras las seis semanas que duró la intervención, se mejoró la capacidad de salto y el tiempo en sprint en los jugadores que participaron en el proceso de intervención. Por el contrario, Ronnestad, Kvamme, Sunde, y Raastad. (2008) no encontraron mejoras en la capacidad de salto ni el tiempo de sprint tras la aplicación durante siete semanas de un programa de fuerza prioritariamente horizontal en futbolistas profesionales. 
Raya-González, J.; Suárez-Arrones, L.; Moreno-Puentedura, M.; Ruiz-Márquez, J., y Sáez de Villareal, E. (2017). Efectos en el rendimiento físico a corto plazo de dos programas de entrenamiento neuromuscular con diferente orientación aplicados en jugadores de fútbol de élite U-17. RICYDE. Revista internacional de ciencias del deporte, 48(13), 88-103.

Gracias a estudios previos conocemos los efectos de diferentes programas de fuerza con mayor orientación vertical $\mathrm{y} / \mathrm{u}$ horizontal sobre las capacidades de rendimiento de los jugadores de fútbol. En cambio, son muy pocas las investigaciones que comparan los efectos de ambos tipos de entrenamiento. Los Arcos, Yanci, Mendiguchia, Salinero, Brughelli, y Castagna. (2014) compararon los efectos de un programa de entrenamiento vertical con otro combinado (vertical + horizontal) demostrando como el programa combinado era más efectivo en la mejora de capacidad de salto y la potencia del tren inferior. Siguiendo esta línea, Ramírez-Campillo, Gallardo, Henriquez-Olguín, Meylan, Martínez, Álvarez, Caniuqueo, Cadore, e Izquierdo. (2015) aplicaron en futbolistas jóvenes un programa pliométrico de diferente orientación (vertical, horizontal o combinado) durante seis semanas, obteniendo mayores mejoras en la capacidad de salto y tiempo de sprint en los jugadores incluidos en el programa combinado. Hasta la fecha aún no se han comparado los efectos de dos programas de entrenamiento neuromuscular (sobrecarga excéntrica + pliometría vs sprint lineal y con cambio de dirección resistidos) sobre el rendimiento en salto, sprint lineal, sprint con cambio de dirección (COD) y potencia muscular del tren inferior en futbolistas jóvenes de alto nivel. Debido a que a ambos métodos de entrenamiento se le presuponen mejoras sobre el rendimiento físico en futbolistas, es necesario comparar sus efectos para obtener información relevante que facilite la posterior inclusión de los mismos en las programaciones de entrenamiento en fútbol.

Por lo tanto, el objetivo de este estudio fue analizar el efecto de dos programas de entrenamiento neuromuscular, con una mayor orientación vertical u horizontal, sobre el rendimiento físico en futbolistas de élite junior.

\section{Método}

\section{Participantes}

Dieciséis futbolistas junior de élite aceptaron voluntariamente participar en este estudio. Los jugadores de fútbol pertenecían al mismo equipo de la cantera de un club de fútbol profesional de $1^{\text {a }}$ División de España, y fueron divididos de forma aleatoria en dos grupos: Grupo Vertical [GV; $\mathrm{n}=8,(16,7 \pm 0,3$ años de edad, 177,1 $\pm 6.5 \mathrm{~cm}$ de altura, $65,9 \pm 5,4 \mathrm{~kg}$ de masa corporal, y $21,1 \pm 1,8 \mathrm{~kg} / \mathrm{m}^{2}$ de índice de masa corporal)] y Grupo Horizontal [GH; $\mathrm{n}=8$, $(16,5 \pm 0,3$ años de edad, $176,9 \pm 7,3 \mathrm{~cm}$ de altura, $66,4 \pm 4,8 \mathrm{~kg}$ de masa corporal, y 21,6 \pm $1,5 \mathrm{~kg} / \mathrm{m}^{2}$ de índice de masa corporal)]. Antes de su participación en el estudio cada jugador completó un cuestionario sobre su historia médica e historial de lesiones. Los porteros fueron excluidos de la muestra de estudio, y los jugadores que no completaron al menos el $80 \%$ de las sesiones de entrenamiento fueron omitidos en el análisis estadístico posterior. Antes de iniciar el estudio, los participantes fueron completamente informados sobre el protocolo a seguir, y el consentimiento escrito e informado fue rellenado por los padres al ser los participantes menores de edad. Todos los participantes eran libres de abandonar el estudio en cualquier momento sin ninguna penalización. Los procedimientos fueron aprobados por el Comité de Ética de la Universidad Pablo de Olavide, Sevilla, (España) y de acuerdo con la Declaración de Helsinki (2013). 
Raya-González, J.; Suárez-Arrones, L.; Moreno-Puentedura, M.; Ruiz-Márquez, J., y Sáez de Villareal, E. (2017). Efectos en el rendimiento físico a corto plazo de dos programas de entrenamiento neuromuscular con diferente orientación aplicados en jugadores de fútbol de élite U-17. RICYDE. Revista internacional de ciencias del deporte, 48(13), 88-103.

\section{Procedimiento}

El estudio fue diseñado para comparar el efecto de un programa de entrenamiento neuromuscular con mayor orientación vertical con otro protocolo de entrenamiento neuromuscular con mayor orientación horizontal. Durante el periodo de intervención, los jugadores siguieron participando en su entrenamiento habitual de fútbol. La rutina semanal consistía en 4 sesiones por semana (martes, miércoles, jueves y viernes) con los siguientes contenidos: desarrollo técnico-táctico, entrenamiento de resistencia, entrenamiento de fuerza, prevención de lesiones y trabajo de flexibilidad $(70 \%, 10 \%, 7 \%, 6 \%$ y $7 \%$ del tiempo total de entrenamiento, respectivamente). Además, durante el tiempo que duró el diseño experimental, los jugadores disputaron nueve partidos de competición (domingo). La intervención duró nueve semanas (entre los meses de enero, febrero y marzo, correspondientes al periodo competitivo). La primera semana se destinó a la familiarización de los jugadores con los dispositivos a utilizar y los test a realizar, en la segunda semana los participantes llevaron a cabo las sesiones del pre-test y en la última semana las sesiones del post-test. En ambas jornadas los futbolistas fueron instruidos para realizar su última comida tres horas antes del comienzo de las pruebas, no tomar bebidas con cafeína, ni realizar ejercicio físico intenso el día de la toma de datos (Sánchez-Sánchez, Hernández, Marcos, Rodríguez y Carretero, 2016). Todos los test se realizaron a la misma hora, entre un gimnasio y el campo de hierba artificial donde el equipo realizaba su entrenamiento, con la indumentaria habitual y el calzado que normalmente usaba el jugador. Los test fueron supervisados por los mismos técnicos especialistas en dos días diferentes, con 48 horas de separación entre cada sesión. El primer día de evaluación, además de realizar el registro de las medidas antropométricas de todos los jugadores, se realizaron los test de salto vertical y el test de media sentadilla. En el segundo día se llevó a cabo el test de sprint lineal y sprint con COD. Antes de cada sesión se realizó un calentamiento estandarizado, que consistió en tres minutos de carrera continua a baja intensidad, ejercicios de movilidad articular y acciones de salto y sprint sobre distancias de 10 a 30m. Los programas de entrenamiento tuvieron una duración de seis semanas y consistieron en dos sesiones semanales (martes y jueves), las cuales se realizaron previamente a la sesión de entrenamiento de fútbol. El entrenamiento del $\mathrm{GV}$, consistió en la combinación de ejercicios realizados en un dispositivo inercial con sobrecarga excéntrica (media sentadilla y squat lateral) y ejercicios pliométricos (salto al cajón) (Tabla 1). El entrenamiento del GH, consistió en ejercicios de sprint lineal y COD de $90^{\circ}$ (dos cambios de dirección en cada repetición, uno a derecha y otro a izquierda) usando un chaleco lastrado con un incremento de peso progresivo en función del porcentaje del peso corporal de cada jugador (Tabla 2). Ambos programas de entrenamiento neuromuscular fueron administrados en base a parámetros similares de volumen e intensidad. Todos los jugadores realizaron un calentamiento estandarizado previo a la sesión específica de entrenamiento neuromuscular, que consistió en una parte general basada en ejercicios aeróbicos y de movilidad, similar para ambos grupos, y una parte específica, en la que se propuso el ejercicio de $1 / 2$ sentadilla bilateral, unilateral con cada pierna y bilateral con salto para el GV; y ejercicios de sprint y COD de $90^{\circ}$ sobre distancias de 10 a $30 \mathrm{~m}$ para el GH. 
Raya-González, J.; Suárez-Arrones, L.; Moreno-Puentedura, M.; Ruiz-Márquez, J., y Sáez de Villareal, E. (2017). Efectos en el rendimiento físico a corto plazo de dos programas de entrenamiento neuromuscular con diferente orientación aplicados en jugadores de fútbol de élite U-17. RICYDE. Revista internacional de ciencias del deporte, 48(13), 88-103.

https://doi.org/10.5232/ricyde2017.04801

Tabla 1. Programa de entrenamiento neuromuscular con mayor orientación vertical.

\begin{tabular}{ccccccc}
\hline Semana & Sesión & Ejercicio 1 & Ejercicio 2 & Intensidad & $\begin{array}{c}\text { Recuperación } \\
\text { entre series }\end{array}$ & Altura \\
\hline 1 & 1 & $1 / 2$ Sentadilla $4 \times(2+6)$ & - & Máxima & $3 \mathrm{~min}$ & - \\
1 & 2 & $1 / 2$ Sentadilla $4 \times(2+6)$ & - & Máxima & $3 \mathrm{~min}$ & - \\
2 & 3 & $1 / 2$ Sentadilla $2 \times(2+8)$ & SL $2 \times(2+6)$ & Máxima & $3 \mathrm{~min}$ & - \\
2 & 4 & $1 / 2$ Sentadilla $2 \times(2+8)$ & SL $2 \times(2+6)$ & Máxima & $3 \mathrm{~min}$ & - \\
3 & 5 & Squat Lateral $4 \times(2+6)$ & - & Máxima & $3 \mathrm{~min}$ & $2 \mathrm{~min}$ \\
3 & 6 & SV a Caja $3 \times 15$ Bilateral & - & Máxima & $2 \mathrm{~cm}$ \\
4 & 7 & SV a Caja $3 \times 20$ Bilateral & - & Máxima & $2 \mathrm{~min}$ & $20 \mathrm{~cm}$ \\
4 & 8 & SV a Caja $4 \times 20$ Bilateral & - & Máxima & $2 \mathrm{~min}$ & $40 \mathrm{~cm}$ \\
5 & 9 & SV a Caja $2 \times 20$ Unilateral & - & Máxima & $2 \mathrm{~min}$ & $20 \mathrm{~cm}$ \\
5 & 10 & SV a Caja $3 \times 20$ Unilateral & - & Máxima & $2 \mathrm{~min}$ & $40 \mathrm{~cm}$ \\
6 & 11 & SV a Caja $2 \times 20$ Unilateral & - & Máxima & $2 \mathrm{~min}$ & $40 \mathrm{~cm}$ \\
6 & 12 & SV a Caja $3 \times 20$ Bilateral & - & Máxima & $2 \mathrm{~min}$ & $60 \mathrm{~cm}$ \\
\hline
\end{tabular}

SV: Salto Vertical; SL: Squat Lateral

Tabla 2. Programa de entrenamiento neuromuscular con mayor orientación horizontal.

\begin{tabular}{cccccccc}
\hline Semana & Sesión & Ejercicio 1 & Series/Reps & Distancia & Intensidad & $\begin{array}{c}\text { Recuperación } \\
\text { entre series }\end{array}$ & $\%$ PC \\
\hline 1 & 1 & COD 15+15+15 & $2 \times 3$ & $45 \mathrm{~m}$ & Máxima & $2 \mathrm{~min} 30 \mathrm{~s}$ & $0 \%$ \\
1 & 2 & Sprint Lineal & $2 \times 3$ & $40 \mathrm{~m}$ & Máxima & $3 \mathrm{~min}$ & $0 \%$ \\
2 & 3 & COD 15+15+15 & $2 \times 4$ & $45 \mathrm{~m}$ & Máxima & $2 \mathrm{~min} 30 \mathrm{~s}$ & $4-5 \%$ \\
2 & 4 & Sprint Lineal & $2 \times 4$ & $40 \mathrm{~m}$ & Máxima & $3 \mathrm{~min}$ & $4-5 \%$ \\
3 & 5 & COD 10+10+10 & $2 \times 5$ & $30 \mathrm{~m}$ & Máxima & 2 min 30s & $7,5-8,5 \%$ \\
3 & 6 & Sprint Lineal & $3 \times 3$ & $30 \mathrm{~m}$ & Máxima & $3 \mathrm{~min}$ & $7,5-8,5 \%$ \\
4 & 7 & COD 10+10+10 & $2 \times 5$ & $30 \mathrm{~m}$ & Máxima & $2 \mathrm{~min} 30 \mathrm{~s}$ & $11-12 \%$ \\
4 & 8 & Sprint Lineal & $3 \times 3$ & $30 \mathrm{~m}$ & Máxima & $3 \mathrm{~min}$ & $11-12 \%$ \\
5 & 9 & COD 5+5+5 & $3 \times 3$ & $15 \mathrm{~m}$ & Máxima & $2 \mathrm{~min} 30 \mathrm{~s}$ & $14-15 \%$ \\
5 & 10 & Sprint Lineal & $4 \times 3$ & $20 \mathrm{~m}$ & Máxima & $3 \mathrm{~min}$ & $14-15 \%$ \\
6 & 11 & COD 5+5+5 & $2 \times 3$ & $15 \mathrm{~m}$ & Máxima & $2 \mathrm{~min} 30 \mathrm{~s}$ & $7,5-8,5 \%$ \\
6 & 12 & Sprint Lineal & $2 \times 3$ & $20 \mathrm{~m}$ & Máxima & $3 \mathrm{~min}$ & $7,5-8,5 \%$ \\
\hline Abreviaturas: COD, Cambio de dirección; PC, peso corporal & & & & \\
\hline
\end{tabular}


Raya-González, J.; Suárez-Arrones, L.; Moreno-Puentedura, M.; Ruiz-Márquez, J., y Sáez de Villareal, E. (2017). Efectos en el rendimiento físico a corto plazo de dos programas de entrenamiento neuromuscular con diferente orientación aplicados en jugadores de fútbol de élite U-17. RICYDE. Revista internacional de ciencias del deporte, 48(13), 88-103.

https://doi.org/10.5232/ricyde2017.04801

\section{Instrumentos y Test}

Antropometría. La altura se midió utilizando un estadímetro de pared (222 Seca; Seca, Nueva York, EE.UU) con una precisión de $2 \mathrm{~mm}$ y un rango de medición de $130-210 \mathrm{~cm}$. La masa corporal se midió utilizando una báscula médica, con una precisión de $0.2 \mathrm{~kg}$ y un rango de medición de 2-130kg (Tanita BC-418 MA; Tanita, Tokyo, Japan).

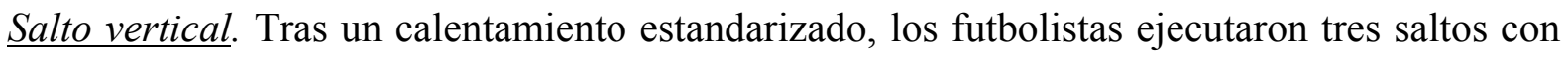
contramovimiento (CMJ) y 3 Abalakov (ABK), utilizando para valorar la altura de cada uno de los saltos una plataforma de rayos infrarrojos (Optojump Next, Microgate ${ }^{\circledR}$, Bolzano, Italia). Durante toda la ejecución del CMJ las manos de los participantes debían estar colocadas en sus caderas, aunque durante la ejecución del ABK se permitió el movimiento de los brazos de manera coordinada y sincronizada con la acción de flexo-extensión de las piernas. Se recomendó a los participantes que realizaran la recepción de cada salto en una posición vertical y que flexionaran las rodillas después del aterrizaje (Sáez de Villarreal, Suárez-Arrones, Requena, Haff y Ferrete, 2015). El mejor valor de cada test fue seleccionado para el posterior análisis estadístico y el tiempo de recuperación propuesto entre cada salto fue de $2 \mathrm{~min}$.

Test de Media Sentadilla. Para el tren inferior se determinó la velocidad media propulsiva (VMP) en un test incremental de cargas en el ejercicio de $1 / 2$ sentadilla (López-Segovia, Palao y González-Badillo, 2010). Los participantes ejecutaron la $1 / 2$ sentadilla desde una posición de inicio completamente extendidos con la barra en contacto con los hombros. A la señal, los participantes ejecutaron la fase excéntrica de manera controlada, seguido y sin pausa, de una extensión de las piernas lo más rápido posible para volver a la posición inicial tras finalizar la fase concéntrica. El tronco debía mantenerse lo más derecho posible, y un experto en entrenamiento de fuerza guio el desarrollo de este test y ayudó a que los participantes realizaran la técnica de manera correcta. Los test fueron realizados con una barra guiada (Máquina Smith ${ }^{\circledR}$; Modelo Adan-Sport, Granada, España) la cual permitía solo movimientos verticales. Se analizó la velocidad media propulsiva gracias a un encoder lineal fijado en la barra. Por medio de su software informático específico se registraron y filtraron los valores obtenidos (T-Force System ${ }^{\circledR}$; Ergotech, Murcia, Spain). Se realizó un calentamiento previo que consistió en una serie de 10 repeticiones con una carga de $15 \mathrm{~kg}$. El test se inició con un peso de $15 \mathrm{~kg}$ en la primera serie y acabó con un peso $60 \mathrm{~kg}$ en la última serie, con pesos de $30 \mathrm{~kg}, 40 \mathrm{~kg}$ y $50 \mathrm{~kg}$ para las series intermedias. En cada serie los participantes ejecutaron 4 repeticiones, y se estableció una recuperación de $5 \mathrm{~min}$ entre cada serie. Para el posterior análisis estadístico, se seleccionó el mejor valor obtenido en base a la VMP en cada una de las cargas utilizadas.

Test de Sprint lineal. Para valorar los tiempos de sprint se emplearon fotocélulas eléctricas (Polifermo Light Radio, Microgate ${ }^{\circledR}$, Bolzano, Italia). Todos los participantes realizaron dos sprints lineales midiendo el tiempo en las distancias de 10, 20 y 30m (Buchheit, MendezVillanueva, Delhomel, Brughelli y Ahmaidi, 2010). En el inicio de cada test el pie delantero se colocó $0,5 \mathrm{~m}$ antes de la primera fotocélula. Se eligió el mejor valor de cada test para el consiguiente análisis estadístico. El tiempo de descanso entre cada repetición fue de $3 \mathrm{~min}$.

Test de Sprint con Cambio de Dirección. Todos los participantes realizaron dos esprines de $20 \mathrm{~m}(10+10 \mathrm{~m})$ con un de COD de $90^{\circ}$ (Figura 1) hacia la derecha (COD-D) y dos esprines con un COD de $90^{\circ}$ hacia la izquierda (COD-I) (Hader, Palazzi y Buchheit, 2015). En el inicio de cada test el pie delantero se colocó $0,5 \mathrm{~m}$ antes de la primera fotocélula (Polifermo Light Radio, Microgate ${ }^{\circledR}$, Bolzano, Italia). Para el posterior análisis estadístico se eligió el 
Raya-González, J.; Suárez-Arrones, L.; Moreno-Puentedura, M.; Ruiz-Márquez, J., y Sáez de Villareal, E. (2017). Efectos en el rendimiento físico a corto plazo de dos programas de entrenamiento neuromuscular con diferente orientación aplicados en jugadores de fútbol de élite U-17. RICYDE. Revista internacional de ciencias del deporte, 48(13), 88-103.

mejor valor de cada test. Se estableció un tiempo de recuperación de 2 min entre repeticiones. El tiempo en el sprint lineal de $20 \mathrm{~m}$ de distancia fue usado con el tiempo en el sprint con cambio de dirección de $20 \mathrm{~m}$ para el cálculo del porcentaje de pérdida de COD.
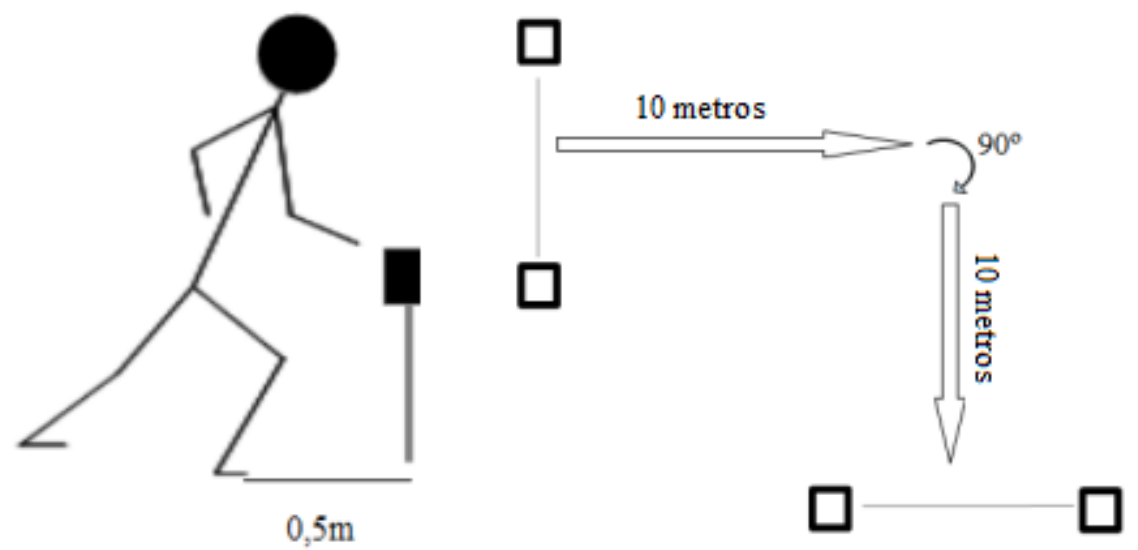

Figura 1. Representación esquemática de la posición de salida y de los cambios de dirección de $90^{\circ}$

\section{Análisis estadístico}

Los datos se presentan como media \pm desviación estándar (DE). Todas las variables objeto de estudio presentaron una distribución normal (test Shapiro-Wilk). El tamaño del efecto [TE, límite de confianza (LC) 90\%] según la propuesta de Cohen (1988) y adaptada por Hopkins, Marshall, Batterham, y Hanin. (2009) fue calculado para comparar la magnitud de las diferencias entre el pre-test y el post-test dentro de cada grupo, al igual que para la comparación entre grupos. Los rangos establecidos para los valores del TE fueron $>0,2$ (pequeño), >0,6 (moderado), y >1,2 (grande) (Hopkins y col., 2009). Las diferencias cuantitativas fueron valoradas de manera cualitativa (VC) (Hopkins y col., 2009) estableciendo las siguientes probabilidades: $<1 \%$, casi seguro que no; $1 \%$ a $5 \%$, muy poco probable; $5 \%$ a $25 \%$, poco probable; $25 \%$ a $75 \%$, posible; $75 \%$ a $95 \%$, probable; $95 \%$ a $99 \%$, muy probable; y $>99 \%$, casi seguro. Se determinó un efecto sustancial a diferencias con una probabilidad $>75 \%$ al igual que en previos estudios (Suárez-Arrones, Tous-Fajardo, Núñez, Gonzalo-Skok, Gálvez, y Méndez-Villanueva, 2014).

\section{Resultados}

No existieron diferencias inter-grupos en ninguna de las variables de rendimiento evaluadas durante el pre-test (Tabla 3). El análisis estadístico intra-grupo mostró en el GV mejoras sustanciales en el CMJ [1,96cm (5,66\%), TE = 0,49, VC: Probable $(93 / 7 / 0 \%)]$, ABK [1,75cm $(4,24 \%), \mathrm{TE}=0,38, \mathrm{VC}$ : Probable $(83 / 16 / 1 \%)]$ y en la VMP con todas las cargas evaluadas en $1 / 2$ sentadilla. También se produjo en este grupo una reducción en el rendimiento en el tiempo de sprint lineal en $10 \mathrm{~m}[0,04 \mathrm{~s}(2,23 \%), \mathrm{TE}=-0,69, \mathrm{VC}$ : Probable $(2 / 8 / 90 \%)], 20 \mathrm{~m}$ $[0,07 \mathrm{~s}(2,32 \%), \mathrm{TE}=-0,86, \mathrm{VC}:$ Muy probable $(1 / 3 / 96 \%)], 30 \mathrm{~m}[0,05 \mathrm{~s}(1,20 \%), \mathrm{TE}=-0,45$, VC: Probable (4/18/78\%)] y en el tiempo de sprint con cambio de dirección de $90^{\circ}$ hacia la izquierda (COD-I) [0,07s (1,95\%), TE = -0,33, VC: Probable (0/6/94\%)]. Además, en el GV tuvo lugar un aumento sustancial de la masa corporal $[0,84 \mathrm{~kg}(1,27 \%)$, TE $=0,14$, VC: 
Raya-González, J.; Suárez-Arrones, L.; Moreno-Puentedura, M.; Ruiz-Márquez, J., y Sáez de Villareal, E. (2017). Efectos en el rendimiento físico a corto plazo de dos programas de entrenamiento neuromuscular con diferente orientación aplicados en jugadores de fútbol de élite U-17. RICYDE. Revista internacional de ciencias del deporte, 48(13), 88-103.

Probable $(89 / 11 / 0 \%)]$ y del índice de masa corporal $\left[0,27 \mathrm{~kg} / \mathrm{m}^{2}(1,28 \%), \mathrm{TE}=0,14, \mathrm{VC}\right.$ : Probable (90/10/0\%)]. Se encontraron mejoras sustanciales en el GH en el tiempo de sprint en $10 \mathrm{~m}[-0,05 \mathrm{~s}(-2,64 \%)$, TE $=0,63$, VC: Muy Probable (98/2/0\%)], en el tiempo de sprint con cambio de dirección de $90^{\circ}$ hacia la derecha (COD-D) $[-0,05 \mathrm{~s}(-1,37 \%)$, TE $=0,36, \mathrm{VC}$ : Probable (76/23/1\%)], en el COD-I [-0,05s (-1,36\%), TE = 0,34, VC: Probable (80/19/1\%)] y en la VMP en algunas de las cargas utilizadas en $1 / 2$ sentadilla. El GH no presentó cambios sustanciales en ninguna de las variables antropométricas estudiadas.

La comparación inter-grupos reflejó diferencias sustanciales (>75\%) en diversas variables del rendimiento (Tabla 4). El GV manifestó mejoras sustancialmente mayores respecto al GH en el CMJ $[\mathrm{TE}=0,39$, VC: Muy Probable (76/22/0\%)], mientras que el GH presentó unas mayores mejoras en el tiempo de sprint en 10m [ES =0,68, VC: Probable (93/7/0\%)], 20m $[\mathrm{ES}=0,72, \mathrm{VC}$ : Muy probable (96/4/0\%)] y 30m [ES =0,57, VC: Probable (88/11/1\%)], y en el tiempo de sprint con cambio de dirección de $90^{\circ}$ hacia la derecha (COD-D) $[\mathrm{ES}=0,73$, VC: Probable (91/8/1\%)] en comparación con el VG. No existieron diferencias inter-grupos en la VMP en ninguna de las cargas utilizadas. 
Raya-González, J.; Suárez-Arrones, L.; Moreno-Puentedura, M.; Ruiz-Márquez, J., y Sáez de Villareal, E. (2017). Efectos en el rendimiento físico a corto plazo de dos programas de entrenamiento neuromuscular con diferente orientación aplicados en jugadores de fútbol de élite U-17. RICYDE. Revista internacional de ciencias del deporte, $48(13), 88-103$.

https://doi.org/10.5232/ricyde2017.04801

Tabla 3. Cambios en el rendimiento después del entrenamiento en ambos grupos, Media \pm SD

\begin{tabular}{|c|c|c|c|c|c|c|c|c|c|c|c|c|}
\hline \multirow[b]{2}{*}{ Variable } & \multicolumn{6}{|c|}{ Grupo Vertical } & \multicolumn{6}{|c|}{ Grupo Horizontal } \\
\hline & Pre-test & Post-test & $\begin{array}{c}\% \\
\text { cambio } \\
\end{array}$ & $\begin{array}{c}\text { TE } \\
(90 \% \mathrm{CL})\end{array}$ & Probabilidad & $\mathrm{VC}$ & Pre-test & Post-test & $\begin{array}{c}\% \\
\text { cambio } \\
\end{array}$ & $\begin{array}{c}\mathrm{TE} \\
(90 \% \mathrm{CL})\end{array}$ & Probabilidad & $\mathrm{VC}$ \\
\hline $\mathrm{CMJ}(\mathrm{cm})$ & $34,60 \pm 3,50$ & $36,56 \pm 3,58$ & 5,66 & $0,49 \pm 0,33$ & $93 / 7 / 0 \%$ & Probable & $35,97 \pm 4,01$ & $36,41 \pm 3,82$ & 1,22 & $0,10 \pm 0,30$ & $28 / 67 / 5 \%$ & Posible \\
\hline Abalakov (cm) & $41,30 \pm 4,40$ & $43,05 \pm 3,22$ & 4,24 & $0,38 \pm 0,33$ & $83 / 16 / 1 \%$ & Probable & $41,88 \pm 3,69$ & $42,46 \pm 3,68$ & 1,38 & $0,14 \pm 0,23$ & $32 / 67 / 1 \%$ & Posible \\
\hline $10 \mathrm{~m}$ sprint $(\mathrm{s})$ & $1,79 \pm 0,06$ & $1,83 \pm 0,03$ & 2,23 & $-0,69 \pm 0,65$ & $2 / 8 / 90 \%$ & Probable & $1,89 \pm 0,08$ & $1,84 \pm 0,06$ & $-2,64$ & $0,63 \pm 0,31$ & $98 / 2 / 0 \%$ & Muy probable \\
\hline 20m sprint (s) & $3,02 \pm 0,07$ & $3,09 \pm 0,05$ & 2,32 & $-0,86 \pm 0,57$ & $1 / 3 / 96 \%$ & Muy probable & $3,16 \pm 0,13$ & $3,13 \pm 0,10$ & $-0,95$ & $0,17 \pm 0,34$ & $44 / 52 / 4 \%$ & Posible \\
\hline $30 \mathrm{~m}$ sprint (s) & $4,20 \pm 0,10$ & $4,25 \pm 0,07$ & 1,20 & $-0,45 \pm 0,58$ & $4 / 18 / 78 \%$ & Probable & $4,38 \pm 0,18$ & $4,33 \pm 0,12$ & $-1,14$ & $0,24 \pm 0,39$ & $58 / 38 / 4 \%$ & Posible \\
\hline COD-D (s) & $3,64 \pm 0,14$ & $3,69 \pm 0,07$ & 1,37 & $-0,33 \pm 0,46$ & $3 / 27 / 70 \%$ & Posible & $3,69 \pm 0,11$ & $3,64 \pm 0,07$ & $-1,37$ & $0,36 \pm 0,49$ & $76 / 23 / 1 \%$ & Probable \\
\hline COD-I (s) & $3,59 \pm 0,11$ & $3,66 \pm 0,05$ & 1,95 & $-0,57 \pm 0,40$ & $0 / 6 / 94 \%$ & Probable & $3,67 \pm 0,13$ & $3,62 \pm 0,10$ & $-1,36$ & $0,34 \pm 0,29$ & $80 / 19 / 1 \%$ & Probable \\
\hline \%Pérdida- COD-D (s) & $0,20 \pm 0,03$ & $0,19 \pm 0,02$ & $-5,01$ & $0,22 \pm 0,53$ & $53 / 38 / 9 \%$ & Posible & $0,17 \pm 0,03$ & $0,16 \pm 0,02$ & $-5,88$ & $0,06 \pm 0,58$ & $33 / 45 / 22 \%$ & Posible \\
\hline \%Pérdida- COD-I (s) & $0,19 \pm 0,02$ & $0,18 \pm 0,03$ & $-5,26$ & $0,12 \pm 0,45$ & $37 / 52 / 11 \%$ & Posible & $0,16 \pm 0,04$ & $0,15 \pm 0,03$ & $-6,25$ & $0,10 \pm 0,42$ & $34 / 55 / 11 \%$ & Posible \\
\hline VMP15 & $1,01 \pm 0,14$ & $1,12 \pm 0,14$ & 10,89 & $0,65 \pm 0,67$ & $88 / 10 / 2 \%$ & Probable & $1,02 \pm 0,08$ & $1,06 \pm 0,11$ & 6,01 & $0,65 \pm 0,68$ & $87 / 10 / 3 \%$ & Probable \\
\hline VMP30 & $0,96 \pm 0,09$ & $1,03 \pm 0,11$ & 7,29 & $0,68 \pm 0,47$ & $96 / 4 / 0 \%$ & Muy probable & $0,89 \pm 0,10$ & $0,94 \pm 0,10$ & 5,62 & $0,46 \pm 0,57$ & $78 / 19 / 3 \%$ & Probable \\
\hline VMP40 & $0,88 \pm 0,09$ & $0,96 \pm 0,12$ & 9,09 & $0,64 \pm 0,60$ & $90 / 9 / 1 \%$ & Probable & $0,85 \pm 0,10$ & $0,87 \pm 0,06$ & 2,35 & $0,25 \pm 0,49$ & $58 / 36 / 6 \%$ & Posible \\
\hline VMP50 & $0,84 \pm 0,08$ & $0,88 \pm 0,09$ & 4,76 & $0,43 \pm 0,47$ & $80 / 18 / 2 \%$ & Probable & $0,77 \pm 0,08$ & $0,80 \pm 0,06$ & 3,90 & $0,44 \pm 0,27$ & $93 / 7 / 0 \%$ & Probable \\
\hline VMP60 & $0,79 \pm 0,07$ & $0,81 \pm 0,05$ & 2,53 & $0,40 \pm 0,37$ & $82 / 17 / 1 \%$ & Probable & $0,73 \pm 0,08$ & $0,74 \pm 0,09$ & 1,37 & $0,16 \pm 0,31$ & $40 / 57 / 3 \%$ & Posible \\
\hline
\end{tabular}

Abreviaturas: CL, intervalos de confianza; TE, tamaño del efecto; VC, evaluación cualitativa; CMJ, salto con contramovimiento,

VMP; velocidad media propulsiva; COD-D, cambio de dirección hacia la derecha; COD-I, cambio de dirección hacia la izquierda 
Raya-González, J.; Suárez-Arrones, L.; Moreno-Puentedura, M.; Ruiz-Márquez, J., y Sáez de Villareal, E. (2017). Efectos en el rendimiento físico a corto plazo de dos programas de entrenamiento neuromuscular con diferente orientación aplicados en jugadores de fútbol de élite U-17. RICYDE. Revista internacional de ciencias del deporte, 48(13), 88-103. https://doi.org/10.5232/ricyde2017.04801

Tabla 4. Diferencias entre GV y GH tras el proceso de entrenamiento, Media \pm SD

\begin{tabular}{|c|c|c|c|c|c|}
\hline Variable & $\%$ GV & $\%$ GH & $\begin{array}{c}\text { TE } \\
(90 \% \text { CL) }\end{array}$ & Probabilidad & VC \\
\hline $\mathrm{CMJ}(\mathrm{cm})$ & $5,66^{*}$ & 1,22 & $0,39 \pm 0,46$ & $76 / 22 / 2 \%$ & Probable \\
\hline Abalakov $(\mathrm{cm})$ & 4,24 & 1,38 & $0,31 \pm 0,43$ & $67 / 30 / 3 \%$ & Posible \\
\hline $10 \mathrm{~m}$ sprint $(\mathrm{s})$ & 2,23 & $-2,64 *$ & $0,68 \pm 0,54$ & $97 / 3 / 0 \%$ & Muy probable \\
\hline $20 \mathrm{~m}$ sprint (s) & 2,32 & $-0,95^{*}$ & $0,72 \pm 0,49$ & $96 / 4 / 0 \%$ & Muy probable \\
\hline $30 \mathrm{~m}$ sprint $(\mathrm{s})$ & 1,20 & $-1,14^{*}$ & $0,57 \pm 0,54$ & $88 / 11 / 1 \%$ & Probable \\
\hline COD-D (s) & 1,37 & $-1,37 *$ & $0,73 \pm 0,67$ & $91 / 8 / 1 \%$ & Probable \\
\hline COD-I (s) & 1,95 & $-1,36$ & $0,92 \pm 0,47$ & $99 / 1 / 0 \%$ & Casi seguro \\
\hline \%Pérdida- COD-D (s) & $-5,01$ & $-5,88$ & $0,12 \pm 0,72$ & $43 / 35 / 22 \%$ & Posible \\
\hline \%Pérdida- COD-I (s) & $-5,26$ & $-6,25$ & $0,05 \pm 0,59$ & $33 / 44 / 23 \%$ & Posible \\
\hline VMP15 & 10,89 & 6,01 & $0,31 \pm 0,99$ & $58 / 23 / 19 \%$ & Posible \\
\hline VMP30 & 7,29 & 5,62 & $0,14 \pm 0,73$ & $45 / 35 / 20 \%$ & Posible \\
\hline VMP40 & 9,09 & 2,35 & $0,37 \pm 0,77$ & $65 / 24 / 11 \%$ & Posible \\
\hline VMP50 & 4,76 & 3,90 & $0,03 \pm 0,51$ & $28 / 50 / 22 \%$ & Posible \\
\hline VMP60 & 2,53 & 1,37 & $0,15 \pm 0,45$ & $42 / 48 / 10 \%$ & Posible \\
\hline
\end{tabular}

Nota: CL, intervalos de confianza; TE, tamaño del efecto; VC, evaluación cualitativa; VMP; velocidad media propulsiva; CMJ, salto con contramovimiento; COD-D, cambio de dirección hacia la derecha; COD-I, cambio de dirección hacia la izquierda; \%GV, diferencias pre-post test en GV; \%GH, diferencias pre-post test en GH *Diferencias sustanciales ( $>75 \%$ ) entre los dos grupos experimentales con mayores mejoras en ese grupo Probabilidad: Porcentaje de posibilidad de tener mejores/similares/peores valores

\section{Discusión}

El objetivo de este estudio fue analizar el efecto de dos programas de entrenamiento neuromuscular, con una mayor orientación vertical u horizontal, en una muestra de jugadores de fútbol de élite junior. Los resultados obtenidos muestran que, tras el periodo de intervención, los jugadores del GV incrementaron su capacidad de salto vertical, medida a través de los test CMJ y ABK, y la VMP estimada mediante el test de $1 / 2$ sentadilla, mientras que empeoraron sus resultados en el tiempo de sprint lineal y COD-I. Sin embargo, los jugadores del GH mejoraron sustancialmente su tiempo en sprint en 10m, COD-I, COD-D así como la VMP con 15-30-50kg.

El CMJ ha ganado importancia en los últimos años por ser un test relevante en el rendimiento en fútbol y útil tanto en la selección de talentos, como para discriminar el nivel competitivo de los futbolistas (Comfort, Stewart, Bloom y Clarkson, 2014), siendo también considerado como uno de los test más fiable para la detección de la fatiga en futbolistas (Gathercole, Sporer, Stellingwerff, y Sleivert, 2015). Los resultados del presente estudio mostraron como el GV presentó mejoras sustanciales en el CMJ $(5,66 \%, \mathrm{TE}=0,49)$. Este resultado parece coincidir con la mayoría de los estudios publicados previamente en los que se examina esta capacidad utilizando programas de entrenamiento similares (Chelly, Ghenem, Abid, Hermassi, Tabka, y Shephard, 2010; De Hoyo, Pozzo, Sañudo, Carrasco, Gonzalo-Skok, Domínguez-Cobo, y MoránCamacho, 2015; Ramírez-Campillo y col., 2015). Sin embargo, también hay que tener en cuenta otra investigación similar aunque con resultados contradictorios (Los Arcos y col., 2014). Estas diferencias entre estudios podrían deberse a la edad de los participantes (jóvenes vs sénior), debido a que el potencial de entrenabilidad de los participantes jóvenes parece influir de manera positiva sobre las mejoras en el 
Raya-González, J.; Suárez-Arrones, L.; Moreno-Puentedura, M.; Ruiz-Márquez, J., y Sáez de Villareal, E. (2017). Efectos en el rendimiento físico a corto plazo de dos programas de entrenamiento neuromuscular con diferente orientación aplicados en jugadores de fútbol de élite U-17. RICYDE. Revista internacional de ciencias del deporte, 48(13), 88-103.

https://doi.org/10.5232/ricyde2017.04801

rendimiento (Michailidis, Fatouros, Primpa, Michailidis, Avloniti, Chatzinikolaou, Barbero-Álvarez, Tsoukas, Douroudos, Draganidis, Leontsini, Margonis, Berberidou, y Kambas, 2013).

Otro test de salto para valorar la fuerza explosiva del tren inferior es el test ABK, para el que se encontraron también cambios sustanciales $(4,24 \%$, TE $=0,38)$ en $\mathrm{GV}$ tras el proceso de entrenamiento. Al igual que en nuestro estudio, Wong y col. (2010) encontraron mejoras sustanciales $(3,3 \%, \mathrm{TE}=0,39)$ en $\mathrm{ABK}$ en jugadores de fútbol junior de élite durante la pretemporada tras un proceso de entrenamiento de fuerza de 12 semanas de duración que incluía una fase de entrenamiento de fuerza compuesta por ejercicios tradicionales y una fase de entrenamiento de potencia en la que se incluyeron ejercicios olímpicos. En el GH no se encontraron mejoras sustanciales respecto a la capacidad de salto vertical, resultados que coinciden con los obtenidos por CamposVázquez, Romero, Toscano-Bendala, León-Prados, Suárez-Arrones y González-Jurado (2015) tras la aplicación de un programa de entrenamiento neuromuscular similar. Por lo tanto, en la comparación inter-grupos el GV obtuvo sustancialmente mejores resultados que el $\mathrm{GH}$ en $\mathrm{CMJ}(\mathrm{TE}=0,39)$. El hecho de que en el programa de entrenamiento propuesto para el GV se hayan incluido ejercicios cuyo patrón de movimiento es similar al que se lleva a cabo en el CMJ, parece ser que ha influido positivamente en los resultados obtenidos por los componentes del GV en este test (De Hoyo y col., 2015).

Diversos estudios presentes en la literatura científica con programas de entrenamiento basados en la aplicación de fuerza vertical presentaron mejoras en el tiempo de sprint (Chelly y col., 2009; Sander y col., 2013). Por el contrario y en base a nuestros resultados, el GV empeoró sustancialmente sus resultados en el tiempo de sprint lineal y con COD-I. Se conoce que el rendimiento en sprint es el producto de la velocidad de zancada y la longitud de zancada, además de numerosos componentes que influyen en esta fórmula aparentemente simple. Debido a que ambos elementos están claramente influenciados por las características antropométricas (Ross, Leveritt, y Riek, 2001), una de las principales explicaciones para la pérdida del rendimiento en el tiempo en sprint observado en el GV podría ser los cambios antropométricos encontrados en los sujetos que componían el GV durante el proceso de entrenamiento. De hecho, tras el post-test el GV aumentó sustancialmente su masa corporal $(1,27 \%, \mathrm{TE}=0,14)$ y su índice de masa corporal $(1,28 \%, \mathrm{TE}=0,14)$ con respecto al pre-test. Por otra parte, tradicionalmente se cree que el rendimiento en las pruebas de sprint dependen en gran medida de factores genéticos, obteniéndose solamente mejoras relativamente pequeñas gracias al efecto del entrenamiento (Ross y col., 2001). Además, para conseguir mayores mejoras en el tiempo de sprint se debe buscar la especificidad en el programa de entrenamiento (Sáez de Villarreal, Suarez-Arrones, Requena, Haff, y Ramos-Veliz, 2014). Es por esto que el GH sí que presentó, tras el proceso de entrenamiento, mejoras sustanciales en el tiempo de sprint lineal en $10 \mathrm{~m}(-2,64 \% \mathrm{ES}=0,63)$ así como en el COD [Derecha $(-1,37 \% \mathrm{TE}=$ $0,36)$ Izquierda $(-1,36 \% \mathrm{TE}=0,34)]$, además de posibles mejoras en el tiempo de sprint en 20 y $30 \mathrm{~m}$. Estos resultados parecen coincidir con los obtenidos en el trabajo de Clark, Stearne, Walts, y Miller. (2010) en el que tras aplicar un programa de entrenamiento de 7 semanas de duración en el que se llevaron a cabo 13 sesiones de sprints resistidos con chaleco lastrado, obtuvieron mejoras significativas en el tiempo en sprint en el intervalo de distancia de 18,3 a 54,9 m (-1,20\% TE =0,25). También existen investigaciones con resultados diferentes, especialmente en distancias más largas 
Raya-González, J.; Suárez-Arrones, L.; Moreno-Puentedura, M.; Ruiz-Márquez, J., y Sáez de Villareal, E. (2017). Efectos en el rendimiento físico a corto plazo de dos programas de entrenamiento neuromuscular con diferente orientación aplicados en jugadores de fútbol de élite U-17. RICYDE. Revista internacional de ciencias del deporte, 48(13), 88-103.

https://doi.org/10.5232/ricyde2017.04801

(Bachero-Mena y González-Badillo, 2014; Iaia, Fiorenza, Perri, Alberti, Millet, y Bangsbo, 2015; Luteberget, Raastad, Seynnes, y Spencer, 2015), diferencias que podrían deberse al dispositivo utilizado, ya que en estos estudios se utilizó un trineo y en nuestro estudio utilizamos un chaleco lastrado como medio para aplicar la resistencia al sprint. Debido a que el GV no presentó mejoras en el tiempo de sprint lineal y COD el GH obtuvo, en el análisis inter-grupos, mejoras sustanciales mayores en el tiempo de sprint en $10 \mathrm{~m}(\mathrm{TE}=0,68), 20 \mathrm{~m}(\mathrm{TE}=0,72)$ y $30 \mathrm{~m}(\mathrm{TE}=0,57)$ así como en el COD-D $(\mathrm{TE}=0,73)$. Estas diferencias inter-grupos parecen estar influenciadas por el aumento sustancial de la masa corporal de los jugadores que formaron parte del GV durante el proceso de intervención (Ross y col., 2001).

En relación con la potencia del tren inferior, nuestros resultados reflejaron mejoras sustanciales en la VMP en el ejercicio de $1 / 2$ sentadilla en ambos grupos. En el GV se obtuvieron mejoras sustanciales respecto al pre-test en todas las cargas utilizadas, un hecho que se podría esperar debido al elevado volumen de ejercicios con orientación vertical y con sobrecarga excéntrica incluidos en la rutina semanal de entrenamiento para este grupo. Estos ejercicios de fuerza con sobrecarga excéntrica se caracterizan por una ejecución más fuerte y rápida del ciclo estiramiento-acortamiento, lo que produce mejoras en la potencia y la fuerza máxima (Sáez de Villarreal y col., 2014). Por otro lado, el GH mejoró sustancialmente en el test de media sentadilla en las cargas de 15$30-50 \mathrm{~kg}$ (Tabla 3), a pesar de que no se incluyeron ejercicios de orientación vertical en su rutina de entrenamiento. Estos resultados no fueron esperados, y podrían demostrar que es posible mejorar la VMP en el ejercicio de $1 / 2$ sentadilla a través de otros ejercicios, aspecto que se fundamenta con los resultados similares encontrados en el trabajo de Campos-Vázquez y col. (2015), y que permite descartar que estas mejoras fueran producidas por el efecto del aprendizaje. A pesar de que el porcentaje de mejora para cada carga utilizada en el test de media sentadilla fue mayor en el GV el análisis inter-grupos no mostró diferencias sustanciales para esta variable en ninguna de las cargas analizadas.

\section{Conclusiones}

La realización de un entrenamiento neuromuscular con mayor orientación vertical (sobrecarga excéntrica y pliometría) produce mejoras en la potencia muscular del tren inferior y en la capacidad de salto, así como una reducción del rendimiento en el tiempo de sprint en 10-20-30m y COD-I; mientras que un entrenamiento neuromuscular con mayor orientación horizontal utilizando un chaleco lastrado mejora el tiempo de sprint lineal y COD y la VMP en futbolistas jóvenes de élite. Además, el análisis inter-grupos demuestra que los efectos producidos por el programa de entrenamiento del GH son superiores al GV en el tiempo de sprint lineal en 10-20-30m y COD-I. Por el contrario, el GV solamente obtuvo mejores resultados respecto al GH en CMJ. A pesar de esto, debemos tener en cuenta que en este estudio se ha utilizado una muestra relativamente pequeña, debido a que los equipos de fútbol de cantera estaban compuestos solamente por 18 jugadores de campo. En vista de los resultados obtenidos, se recomienda para futuras investigaciones realizadas con jugadores jóvenes de fútbol llevar a cabo un control del estado antropométrico de los jugadores en intervalos cortos de tiempo. 
Raya-González, J.; Suárez-Arrones, L.; Moreno-Puentedura, M.; Ruiz-Márquez, J., y Sáez de Villareal, E. (2017). Efectos en el rendimiento físico a corto plazo de dos programas de entrenamiento neuromuscular con diferente orientación aplicados en jugadores de fútbol de élite U-17. RICYDE. Revista internacional de ciencias del deporte, 48(13), 88-103.

https://doi.org/10.5232/ricyde2017.04801

Según los resultados obtenidos en el presente estudio, el programa de entrenamiento del GH parece ser más efectivo para la optimización del rendimiento físico en jugadores de fútbol jóvenes de alto nivel. Sin embargo, es imprescindible conocer los efectos y limitaciones de cada programa de entrenamiento para así poder elegir el más adecuado a las necesidades de cada deportista con el fin de optimizar su rendimiento físicodeportivo.

\section{Referencias}

Bachero-Mena, B., \& González-Badillo, J.J. (2014). Effects of resisted sprint training on acceleration with three different loads accounting for $5,12.5$, and $20 \%$ of body mass. Journal of Strength and Conditioning Research, 28, 2954-2960. https://doi.org/10.1519/JSC

Buchheit, M.; Mendez-Villanueva, A.; Delhomel, G.; Brughelli, M., \& Ahmaidi, S. (2010). Improving repeated sprint ability in young elite soccer players: repeated shuttle sprint vs explosive strength training. Journal of Strength and Conditioning Research, 24, 2715-2722. https://doi.org/10.1519/JSC

Bush, M.; Barnes, C.; Archer, D.T.; Hogg, B., \& Bradley, P.S. (2015). Evolution of match performance parameters for various playing positions in the English Premier League. Human Movement Science, 39, 1-11. https://doi.org/10.1016/j.humov.2014.10.003

Campos-Vazquez, M.A.; Romero-Boza, S.; Toscano-Bendala, F.J.; Leon-Prados, J.A.; Suarez-Arrones, L.J., \& Gonzalez-Jurado, J.A. (2015). Comparison of the effect of repeated-sprint training combined with two different methods of strength training on young soccer players. Journal of Strength and Conditioning Research, 29(3), 744-751. https://doi.org/10.1519/JSC.0000000000000700

Castagna, C.; D'Ottavio, S., \& Abt, G. (2003). Activity profile of young soccer players during actual match play. Journal of Strength and Conditioning Research, $17,775-780$.

Chelly, M.S.; Fathloun, M.; Cherif, N.; Ben Amar, M.; Tabka, Z., \& Van Praagh, E. (2009). Effects of a back squat training program on leg power, jump, and sprint performances in junior soccer players. Journal of Strength and Conditioning Research, 23(8), 2241-2249. https://doi.org/10.1519/JSC.0b013e3181b86c40

Chelly, M.S.; Ghenem, M.A.; Abid, K.; Hermassi, S.; Tabka, Z., \& Shephard, R.J. (2010). Effects of in-season short-term plyometric training program on leg power, jump and sprint performance of soccer players. Journal of Strength and Conditioning Research, 24(10), 2670-2676. https://doi.org/10.1519/JSC.0b013e3181e2728f

Clark, K.P.; Stearne, D.J.; Walts, C.T., \& Miller, A.D. (2010). The longitudinal effects of resisted sprint training using weighted sleds vs. weighted vests. Journal of Strength and Conditioning Research, 24, 3287-3295. https://doi.org/10.1519/JSC.0b013e3181b62c0a

Cohen, J. (1988). Statistical power analysis for the behavioral sciences (2nd ed.). Hillsdale, NJ: Lawrence Earlbaum Associates. 
Raya-González, J.; Suárez-Arrones, L.; Moreno-Puentedura, M.; Ruiz-Márquez, J., y Sáez de Villareal, E. (2017). Efectos en el rendimiento físico a corto plazo de dos programas de entrenamiento neuromuscular con diferente orientación aplicados en jugadores de fútbol de élite U-17. RICYDE. Revista internacional de ciencias del deporte, 48(13), 88-103.

https://doi.org/10.5232/ricyde2017.04801

Comfort, P.; Stewart, A.; Bloom, L., \& Clarkson, B. Relationships between strength, sprint, and jump performance in well-trained youth soccer players. (2014). Journal of Strength and Conditioning Research, 28, 173-177.

https://doi.org/10.1519/JSC.0b013e318291b8c7

Cronin, J. B., \& Hansen, K. T. (2005). Strength and power predictors of sports speed. Journal of Strength and Conditioning Research, 19(2), 349-357.

De Hoyo, M.; Pozzo, M.; Sañudo, B.; Carrasco, L.; Gonzalo-Skok, O.; DomínguezCobo, S., \& Morán-Camacho, E. (2015). Effects of a 10-week in-season eccentric-overload training program on muscle-injury prevention and performance in junior elite soccer players. International Journal of Sports Physiology and Performance, 10(1), 46-52. https://doi.org/10.1123/ijspp.2013-0547

Di Salvo, V.; Baron, R.; Tschan, H.; Calderon-Montero, F. J.; Bachl, N., \& Pigozzi, F. (2007). Performance characteristics according to playing position in elite soccer. International Journal of Sports Medicine, 28(3), 222-227.

Gorostiaga, E.; Izquierdo, M.; Ruesta, M.; Iribarren, J.; González-Badillo, J.J., \& Ibáñez, J. (2004). Strength training effects on physical performance and serum hormones in young soccer players. European Journal of Applied Physiology, 91, 698-707.

Gathercole, R.J.; Sporer, B.C.; Stellingwerff, T., \& Sleivert, G.G. (2015). Comparison of the Capacity of Different Jump and Sprint Field Tests to Detect Neuromuscular Fatigue. Journal of Strength and Conditioning Research, 29, 2522-2531. https://doi.org/10.1519/JSC.0000000000000912

Hader, K.; Palazzi, D., \& Buchheit, M. (2015). Change of direction speed in soccer: How much braking is enough? Kinesiology, 47(1), 67-47.

Hoff, J., \& Helgerud, J. (2004). Endurance and strength training for soccer players: physiological considerations. Sports Medicine, 34, 165-180.

Hopkins, W.G.; Marshall, S.W.; Batterham, A.M., \& Hanin, J. (2009). Progressive statistics for studies in sports medicine and exercise science. Medicine \& Science in Sports \& Exercise, 41, 3-13. https://doi.org/10.1249/MSS.0b013e31818cb278

Iaia, F.M.; Fiorenza, M.; Perri, E.; Alberti, G.; Millet, G.P., \& Bangsbo, J. (2015). The Effect of Two Speed Endurance Training Regimes on Performance of Soccer Players. PLoS One, 10,(9)e0138096.

https://doi.org/10.1371/journal.pone.0138096

López-Segovia, M.; Palao, J.M., \& González-Badillo, J.J. (2010). Effect of 4 months of training on aerobic power, strength, and acceleration in two under-19 soccer teams. Journal of Strength and Conditioning Research, 19 (10), 2705-2714. https://doi.org/10.1519/JSC.0b013e3181cc237d

Los Arcos, A.; Yanci, J.; Mendiguchia, J.; Salinero, J.J.; Brughelli, M., \& Castagna, C. (2014). Short-term training effects of vertically and horizontally oriented exercises on neuromuscular performance in professional soccer players. International Journal of Sports Physiology and Performance, 9(3), 480-488. https://doi.org/10.1123/ijspp.2013-0063 
Raya-González, J.; Suárez-Arrones, L.; Moreno-Puentedura, M.; Ruiz-Márquez, J., y Sáez de Villareal, E. (2017). Efectos en el rendimiento físico a corto plazo de dos programas de entrenamiento neuromuscular con diferente orientación aplicados en jugadores de fútbol de élite U-17. RICYDE. Revista internacional de ciencias del deporte, 48(13), 88-103.

https://doi.org/10.5232/ricyde2017.04801

Luteberget, L.S.; Raastad, T.; Seynnes, O., \& Spencer, M. (2015). Effect of traditional and resisted sprint training in highly trained female team handball players. International Journal of Sports Physiology and Performance, 10, 642647.

https://doi.org/10.1123/ijspp.2014-0276

Marques, M.C.; Pereira, A.; Reis, I.G., \& van den Tillaar, R. (2013). Does an inSeason 6-Week Combined Sprint and Jump Training Program Improve StrengthSpeed Abilities and Kicking Performance in Young Soccer Players? Journal of Human Kinetics, 31(39), 157-166.

https://doi.org/10.2478/hukin-2013-0078

Maulder, P., \& Cronin, J. (2005). Horizontal and vertical jump assessment: Reliability, symmetry, discriminative and predictive ability. Physical Therapy in Sport, 6, 74-82.

Meylan, C., \& Malatesta, D. (2009). Effects of in-season plyometric training within soccer practice on explosive actions of young players. Journal of Strength and Conditioning Research, 23(9), 2605-2613. https://doi.org/10.1519/JSC.0b013e3181b1f330

Michailidis, Y.; Fatouros, I.G.; Primpa, E.; Michailidis, C.; Avloniti, A.; Chatzinikolaou, A.; Barbero-Álvarez, J.C.; Tsoukas, D.; Douroudos, I.I.; Draganidis, D.; Leontsini, D.; Margonis, K.; Berberidou, F., \& Kambas, A. (2013). Plyometrics' trainability in pre-adolescent soccer athletes. Journal of Strength and Conditioning Research, 27(1), 38-49. https://doi.org/10.1519/JSC.0b013e3182541ec6

Ramírez-Campillo, R.; Gallardo, F.; Henriquez-Olguín, C.; Meylan, C.M.; Martínez, C.; Álvarez, C.; Caniuqueo, A.; Cadore, E.L., \& Izquierdo, M. (2015). Effect of Vertical, Horizontal, and Combined Plyometric Training on Explosive, Balance, and Endurance Performance of Young Soccer Players. Journal of Strength and Conditioning Research, 29(7), 1784-1795. https://doi.org/10.1519/JSC.0000000000000827

Reilly, T.; Bangsbo, J., \& Franks, A. (2000). Anthropometric and physiological predispositions for elite soccer. Journal of Sports Sciences, 18, 669-683.

Ronnestad, B.R.; Kvamme, N.H.; Sunde, A., \& Raastad, T. (2008). Short-term effects of strength and plyometric training on sprint and jump performance in professional soccer players. Journal of Strength and Conditioning Research, 22(3), 773-780.

https://doi.org/10.1519/JSC.0b013e31816a5e86

Ross, A.; Leveritt, M., \& Riek, S. (2001). Neural influences on sprint running. Training adaptations and acute responses. Sports Medicine, 31, 409-425.

Sáez de Villarreal, E.; Suarez-Arrones, L.; Requena, B.; Haff, G.G, \& Ramos-Veliz, R. (2014). Effects of dry-land vs. in-water specific strength training on professional male water polo players' performance. Journal of Strength and Conditioning Research, 28, 3179-3188. https://doi.org/10.1519/JSC.0000000000000514

Sáez de Villarreal, E.; Suarez-Arrones, L.; Requena, B.; Haff, G.G, \& Ferrete, C. (2015). Effects of Plyometric and Sprint Training on Physical and Technical Skill Performance in Adolescent Soccer Players. Journal of Strength and Conditioning Research, 29(7), 1894-1903.

https://doi.org/10.1519/JSC.0000000000000838 
Raya-González, J.; Suárez-Arrones, L.; Moreno-Puentedura, M.; Ruiz-Márquez, J., y Sáez de Villareal, E. (2017). Efectos en el rendimiento físico a corto plazo de dos programas de entrenamiento neuromuscular con diferente orientación aplicados en jugadores de fútbol de élite U-17. RICYDE. Revista internacional de ciencias del deporte, 48(13), 88-103.

https://doi.org/10.5232/ricyde2017.04801

Sánchez-Sánchez, J.; Hernández, C.; Marcos, V.; González, A., \& Carretero, M. (2016). Efecto de un entrenamiento intermitente con y sin cambios de dirección, sobre el rendimiento físico de jóvenes futbolistas. RETOS. Nuevas Tendencias en Educación Física, Deporte y Recreación, 30, 70-75.

Sander, A.; Keiner, M.; Wirth, K., \& Schmidtbleicher, D. (2013). Influence of a 2year strength training programme on power performance in elite youth soccer players. European Journal of Sport Sciences, 13(5), 445-451.

https://doi.org/10.1080/17461391.2012.742572

Suarez-Arrones, L.; Tous-Fajardo, J.; Núñez, J.; Gonzalo-Skok, O.; Gálvez, J., \& Méndez-Villanueva, A. (2014). Concurrent repeated-sprint and resistance training with superimposed vibrations in rugby players. International Journal of Sports Physiology and Performance, 9(4), 667-673. https://doi.org/10.1123/ijspp.2013-0238

Thomas, K.; French, D., \& Hayes, P.R. (2009). The effect of two plyometric training techniques on muscular power and agility in youth soccer players. Journal of Strength and Conditioning Research, 23(1), 332-335. https://doi.org/10.1519/JSC.0b013e318183a01a

Wong, P.L.; Chamari, K., \& Wisløff, U. (2010). Effects of 12-week on-field combined strength and power training on physical performance among U-14 young soccer players. Journal of Strength and Conditioning Research, 24, 644-652.

https://doi.org/10.1519/JSC.0b013e3181ad3349 